\title{
On Some Dynamics of Duffing Dynamical System Generated by a Semi-Discretization Process with Two Different Delays
}

\author{
Ahmed M. A. El-Sayed ${ }^{1}$, Zaki F. El-Raheem ${ }^{2}$ and Sanaa M. Salman ${ }^{2, *}$ \\ ${ }^{1}$ Mathematics and computer Science department, Faculty of Science, Alexandria University, Alexandria, Egypt \\ ${ }^{2}$ Mathematics department, Faculty of Education, Alexandria University, Alexandria, Egypt
}

Received: 13 Nov. 2013, Revised: 17 Feb. 2014, Accepted: 18 Feb. 2014

Published online: 1 May 2014

\begin{abstract}
In this paper, a semi-discretization process is applied to the Duffing system to obtain its continuous time difference equations counterpart with two different delays. Fixed points and their asymptotic stability of the resulting system are investigated. Bifurcation and chaos are discussed.
\end{abstract}

Keywords: Continuous time difference equations , semi-discretization process, Duffing system, fixed points, stability, bifurcation, chaos.

\section{Introduction}

A new class of discontinuous (sectionally continuous) dynamical systems generated by a semi-discretization process has been defined in [14]. Here we are concerned with the Duffing system in the unforced case given by

$$
\begin{aligned}
& \frac{d x}{d t}=y, \\
& \frac{d y}{d t}=-k y-x\left(1+x^{2}\right),
\end{aligned}
$$

where $k$ is the damping coefficient [12]. The solution of this system has been studied in many papers and its hopf bifurcation was analyzed as well. In this work, we are interested in the discontinuous (sectionally continuous) counterpart of this system.

Consider the problem of retarded functional equation

$$
x(t)=f(x(t-r)), \quad t \in(0, T],
$$

with the initial condition

$$
x(\tau)=\phi(\tau), \quad \tau \leq 0 .
$$

If $T$ is a positive integer, $r=1, \phi(0)=x_{o}$ and $t=n=1,2,3, \ldots$, then the problem (1)-(2) will be the discrete dynamical system

$$
\begin{gathered}
x_{n}=f\left(n, x_{n-1}\right), \quad n=1,2,3, \ldots, T \\
x(0)=x_{o} .
\end{gathered}
$$

This shows that the discrete dynamical system (3)-(4) is a special case of the problem of the retarded functional equation (1)-(2).

Let $t \in(0, r]$, then $t-r \in(-r, 0]$ and the solution of (1)-(2) is given by

$$
x(t)=x_{1}(t)=f(\phi(0)), \quad t \in(0, r] .
$$

For $t \in(r, 2 r]$, then $t-r \in(0, r]$ and the solution of (1) is given by

$x(t)=x_{2}(t)=f\left(x_{1}(t)\right)=f(f(\phi(0)))=f^{2}(\phi(0)), \quad t \in(r, 2 r]$.

Repeating the process we can easily deduce that the solution of (1) is given by

$$
x(t)=x_{n}(t)=f^{n}(\phi(0)), \quad t \in((n-1) r, n r],
$$

which is continuous on each subinterval $((k-1) r, k r], k=$ $1,2,3, \ldots, n$, but

$$
\lim _{t \rightarrow k r^{+}} x_{(k+1) r}(t)=f^{k+1}(\phi(0)) \neq x_{k r}
$$

\footnotetext{
*Corresponding author e-mail: findingsanaa@yahoo.com
} 
which implies that the solution of the problem (1)-(2) is discontinuous (sectionally continuous) on $(0, T]$ and thus we have proved the following theorem [7].

Theorem 1.The solution of the problem of retarded functional equation (1)-(2) is discontinuous (sectionally continuous) even if the functions $f$ and $\phi$ are continuous.

\section{A semi-discretization process}

Consider the Duffing system given by

$$
\begin{aligned}
& \frac{d x}{d t}=y, \\
& \frac{d y}{d t}=-k y-x\left(1+x^{2}\right) .
\end{aligned}
$$

The discontinuous (sectionally continuous) counterpart of this system may be obtained by applying a semi-discretization process as follows

Let $r>0$ be given. Using The approximations

$$
\begin{aligned}
& x^{\prime}(t) \simeq \frac{x\left(t+r_{1}\right)-x(t)}{r_{1}}, \\
& y^{\prime}(t) \simeq \frac{y\left(t+r_{2}\right)-y(t)}{r_{2}},
\end{aligned}
$$

in $(5)$ we get

$$
\begin{aligned}
\frac{x\left(t+r_{1}\right)-x(t)}{r_{1}} & \simeq y, \\
x(t) & =x\left(t-r_{1}\right)+r_{1} y\left(t-r_{1}\right) .
\end{aligned}
$$

Similarly,

$$
\begin{aligned}
\frac{y\left(t+r_{2}\right)-y(t)}{r_{2}} & \simeq-k y-x\left(1+x^{2}\right), \\
y(t) & =\left(1-k r_{2}\right) y\left(t-r_{2}\right)-r_{2} x\left(t-r_{2}\right)\left(1+x^{2}\left(t-r_{2}\right)\right) .
\end{aligned}
$$

That is, we have the following discontinuous (sectionally continuous) counterpart of the Duffing system

$x(t)=x\left(t-r_{1}\right)+r y\left(t-r_{1}\right), \quad t \in(0, T]$,

$y(t)=\left(1-k r_{2}\right) y\left(t-r_{2}\right)-r_{2} x\left(t-r_{2}\right)\left(1+x^{2}\left(t-r_{2}\right)\right)$.

with the initial conditions

$x(0)=x_{o}, \quad t \leq 0$,

$y(0)=y_{o}, \quad t \leq 0$.

By the same way as in section 2, we can show that the solution of system (7)-(8) for $t \in(n r,(n+1) r]$ is given by the loop

$x_{n+1}(t)=x_{n}\left(n r_{1}\right)+r_{1} y_{n}\left(n r_{1}\right), \quad n=1,2,3, \ldots$

$y_{n+1}(t)=\left(1-k r_{2}\right) y_{n}\left(n r_{2}\right)-r_{2} x_{n}\left(n r_{2}\right)\left(1+x_{n}^{2}\left(n r_{2}\right)\right)$.

It is worth to mention here that the previous semi-discretization process can be obtained by Taylor

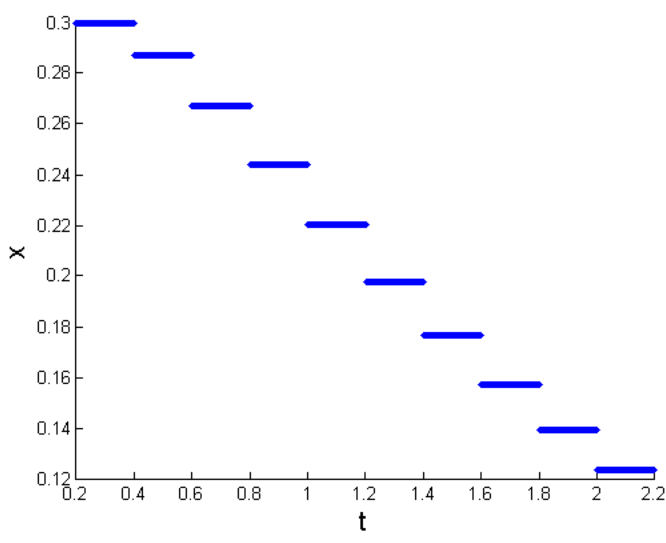

Fig. 1: Trajectory of (7)-(8) with $k=2.36$ and $r_{1}=r_{2}=0.3$

expansion as follows:

$$
\begin{aligned}
x(t) & =x\left(t-r_{1}\right)+x^{\prime}\left(t-r_{1}\right) \frac{t-\left(t-r_{1}\right)}{1 !}+\ldots \\
& \simeq x\left(t-r_{1}\right)+r_{1} y\left(t-r_{1}\right) .
\end{aligned}
$$

The same thing can be said to the second equation.

We should explain here the main difference between our new approach which is called a semi-discretization process and the well-known Euler's method. Indeed, Euler's method neglects the step size " $h$ " which we do consider and rename it $r$, the delay parameter. The reason is that $r$ plays a very important rule in the discretization process and as we will see below the stability of the fixed points depends on it. In addition, bifurcation, chaos, and chaotic attractor depend on it.

To summarize, we showed that there exists a semi-discretization process which generate a discontinuous (sectionally continuous) dynamical system. This discontinuous (sectionally continuous) dynamical system generalizes the discrete one studied in [5].

Figures (1)and (2) show the trajectory of the discontinuous (sectionally continuous) system (7)-(8) when $r_{1}=r_{2}=1$, while Figure (3) shows the trajectory of the continuous system (5)-(6).

\subsection{Approximate Solution}

In this part we show that the proposed semi-discretization process best approximates the solution of the discontinuous (sectionally continuous) dynamical system (7)-(8) to the exact solution of the continuous system (5)-(6) as shown in the table below. Now for $t=\frac{n+n+1}{2} r$, the following table gives the absolute error $=$ ||exact-approximate $\|$ for some different values of $n$ and $r$. Here we take the 2-norm defined by $\|.\|_{2}=\sqrt{\left(x_{\text {approximate }}-x_{\text {exact }}\right)^{2}+\left(y_{\text {approximate }}-y_{\text {exact }}\right)^{2}}$. 


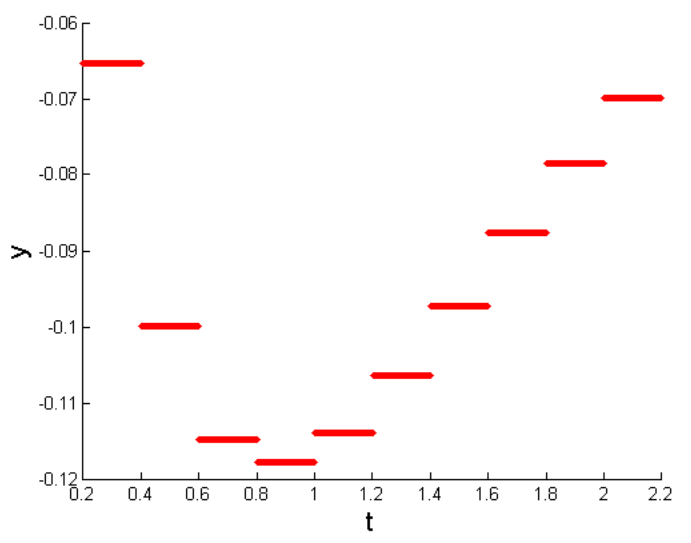

Fig. 2: Trajectory of (7)-(8) with $k=2.36$ and $r_{1}=r_{2}=0.3$
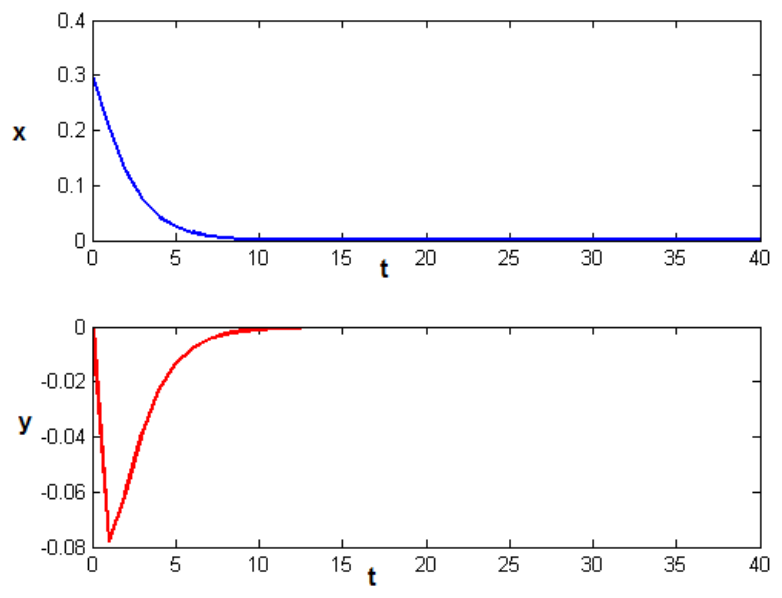

Fig. 3: Trajectory of (5)-(6) with $k=2.36$

Table 1: Absolute error

\begin{tabular}{cccc}
\hline \hline $\mathrm{n}$ & $\mathrm{r}=0.1$ & $\mathrm{r}=0.2$ & $\mathrm{r}=0.3$ \\
\hline \hline 10 & 0.8924451086 & 0.10550839949793 & 0.1071 \\
20 & 0.0463050773 & 0.03078535254359 & 0.0140 \\
30 & 0.02603177630 & 0.00829843070025 & 0.0018 \\
40 & 0.0134458198 & 0.00222970626991 & 0.7673 \\
50 & 0.0070740497 & $5.808440584525678 \mathrm{e}-004$ & 0.1092 \\
\hline
\end{tabular}

\section{Fixed points and stability}

The discontinuous (sectionally continuous) dynamical system (7)-(8) has three fixed points namely, $(0,0),(i, 0)$ and $(-i, 0)$ which can be easily obtained by solving the system

$x=x+r y$,

$y=(1-k r) y-r x\left(1+x^{2}\right)$.
To determine the stability of a fixed point, consider a small perturbation from the fixed point by letting

$$
x(t)=x_{f i x}+\varepsilon_{0} \lambda^{t} .
$$

and

$$
y(t)=y_{f i x}+\eta_{0} \lambda^{t}
$$

Substituting in (7)-(8), we get

$$
\begin{gathered}
x_{f i x}+\varepsilon_{0} \lambda^{t}=x_{f i x}+\varepsilon_{0} \lambda^{t-r_{1}}+r_{1}\left(y_{f i x}+\eta_{0} \lambda^{t}\right), \\
y_{f i x}+\eta_{0} \lambda^{t}=\left(1-k r_{2}\right)\left(y_{f i x}+\eta_{0} \lambda^{t-r_{2}}\right)-r_{2}\left(x_{f i x}+\right. \\
\left.\varepsilon_{0} \lambda^{t-r_{2}}\right)\left(1+\left(x_{f i x}+\varepsilon_{0} \lambda^{t-r_{2}}\right)^{2}\right) .
\end{gathered}
$$

Expanding the right hand side for each equation using Taylor expansion and neglecting higher orders we get

$$
\begin{gathered}
1=\lambda^{-r_{1}}, \\
1=\left(1-k r_{2}\right) \lambda^{-r_{2}}
\end{gathered}
$$

So, we conclude that the fixed points are asymptotically stable if all roots of the equations (11) and (12) satisfy $|\lambda|<1$.

\section{Bifurcation and chaos}

In this section we show by numerical experiments illustrated by bifurcation diagrams that the dynamical behaviors of the discontinuous (sectionally continuous) dynamical system (7)-(8) are completely affected by the change in both $r$ and $T$. We take $k=2.36$ in all numerical simulations showing attractors. We are considering three cases for different delays $r_{1}$ and $r_{2}$ as follows

- Case1: $r_{1}=r_{2}=r$

Let $r_{1}=r_{2}=r$ and vary $k$ from 2 to 3 with step length 0.001 . The initial state of the system is $\left(x_{0}, y_{0}\right)=(0.1,0)$. The period doubling bifurcation occurs at $k=2.8$ while its occurrence is delayed to $k=3$ when $r$ is decreased. Figures from (4) to (16) illustrate the relation between $r$ and the occurrence of the period doubling bifurcation, it's a reversal relation, when $r$ is decreased, the period doubling bifurcation shows after some delay.

Take $r=1$ and $t \in[0,300]$ in (7)-(8) (Figures (4),(5)).

Take $r=0.9$ and $t \in[0,120]$ in (7)(8) (Figure (6),(7)).

Take $r=0.8$ and $t \in[0,300]$ in (7)-(8) (Figure (8),(9)).

Take $r=1.1$ and $t \in[0,300]$ in (7)-(8) (Figure (10),(11)).

Take $r=0.1$ and $t \in[0,150]$ in (7)-(8) (Figure (12),(13)).

Take $r=1.2$ and $t \in[0,300]$ in (7)-(8) (Figure (14),(15)).

Take $r=0.5$ and $t \in[0,300]$ in (7)-(8) (Figure (16),(17)).

- Case 2: $r_{1}>r_{2}$

Now let $r_{1}>r_{2}$ and vary $k$ from 8 to 10 with step length 0.001 . The initial state of the system is the same as above 


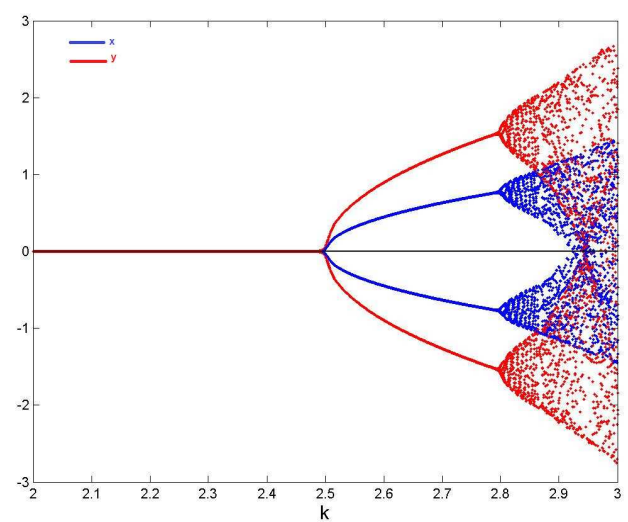

Fig. 4: Bifurcation diagram of (7)-(8) when $r_{1}=r_{2}=1$ and $t \in$ $[0,300]$.

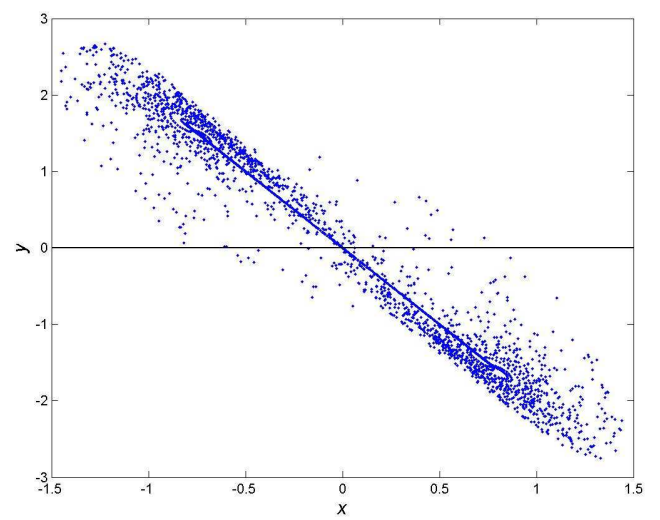

Fig. 5: Chaotic attractor of (7)-(8) when $r_{1}=r_{2}=1$ and $t \in$ $[0,300]$.

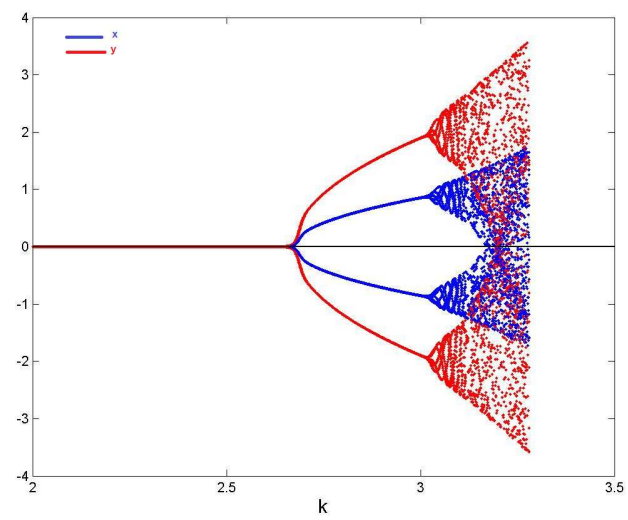

Fig. 6: Bifurcation diagram of (7)-(8) when $r_{1}=r_{2}=0.9$ and $t \in[0,120]$.

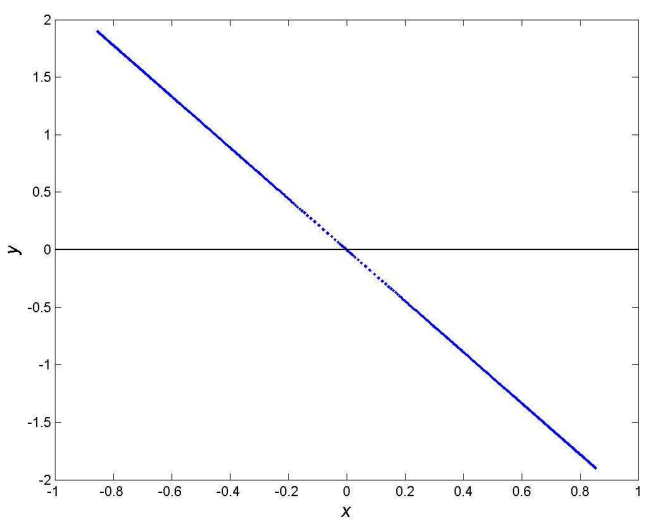

Fig. 7: Chaotic attractor of (7)-(8) when $r_{1}=r_{2}=0.9$ and $t \in$ $[0,120]$.

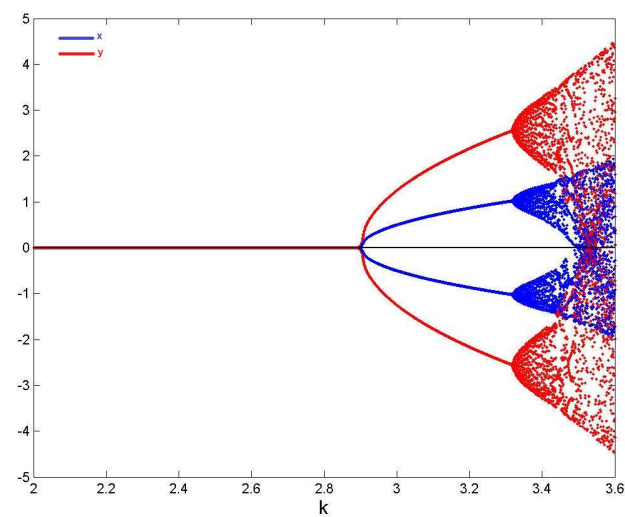

Fig. 8: Bifurcation diagram of (7)-(8) when $r_{1}=r_{2}=0.8$ and $t \in[0,300]$.

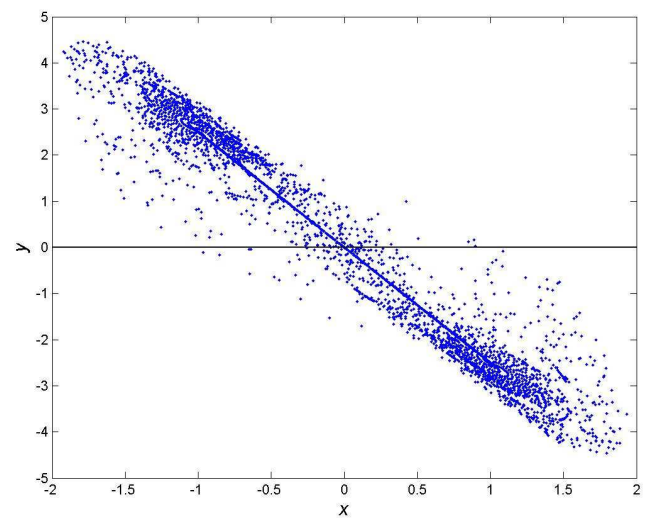

Fig. 9: Chaotic attractor of (7)-(8) when $r_{1}=r_{2}=0.8$ and $t \in$ $[0,300]$. 


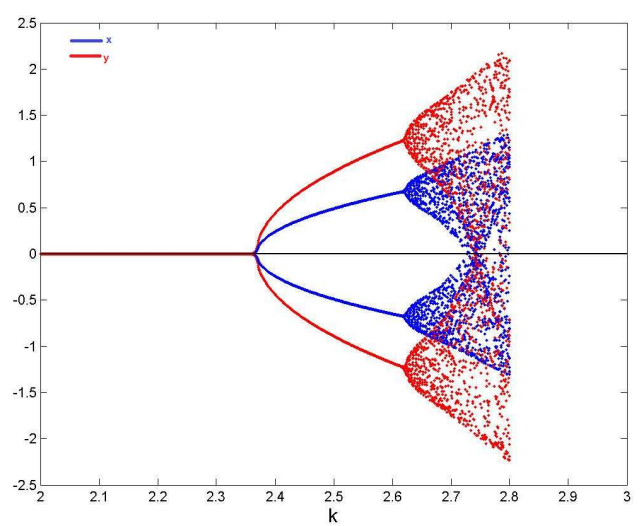

Fig. 10: Bifurcation diagram of (7)-(8) when $r_{1}=r_{2}=1.1$ and $t \in[0,300]$.

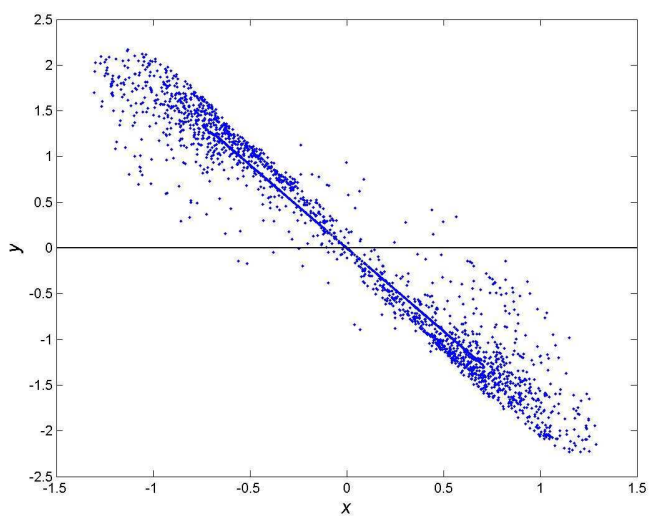

Fig. 11: Chaotic attractor of (7)-(8) when $r_{1}=r_{2}=1.1$ and $t \in$ $[0,300]$.

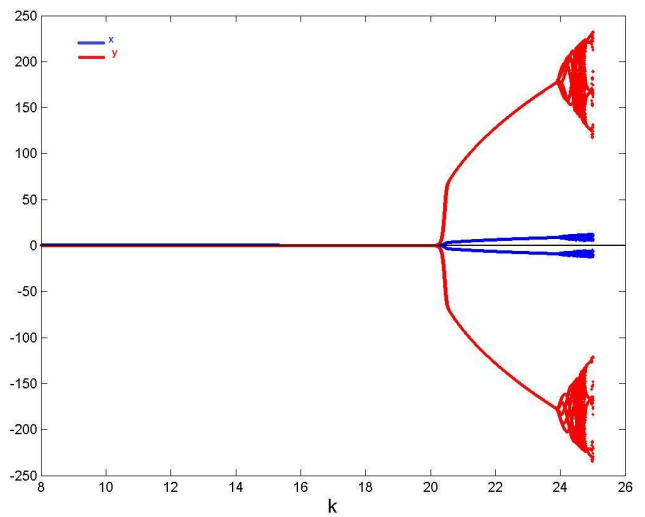

Fig. 12: Bifurcation diagram of (7)-(8) when $r_{1}=r_{2}=0.1$ and $t \in[0,150]$.

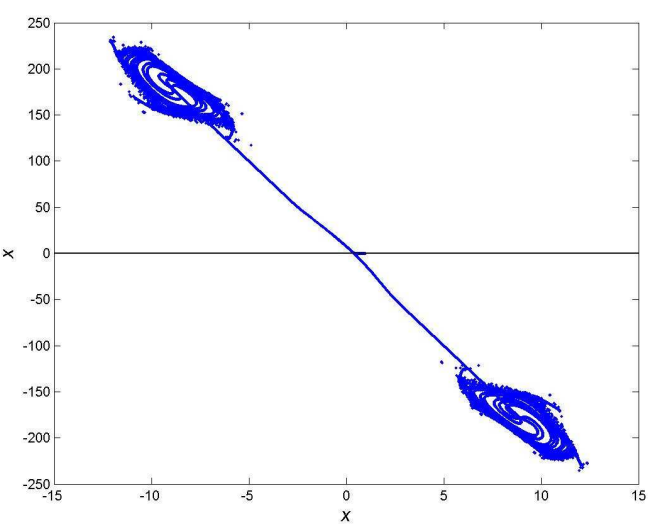

Fig. 13: Chaotic attractor of (7)-(8) when $r_{1}=r_{2}=0.1$ and $t \in$ $[0,150]$.

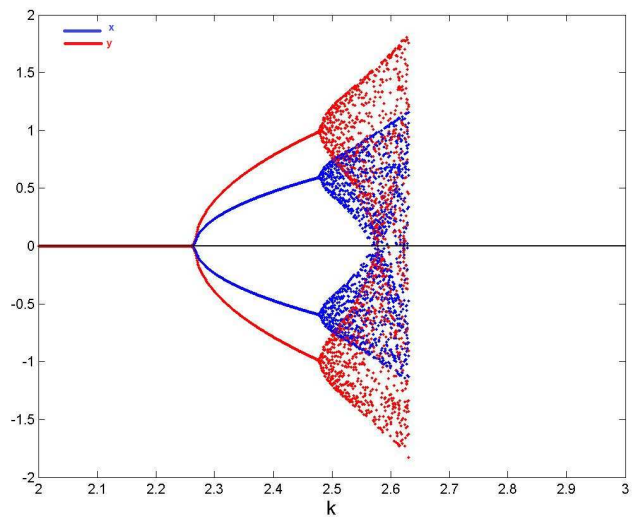

Fig. 14: Bifurcation diagram of (7)-(8) when $r_{1}=r_{2}=1.2$ and $t \in[0,300]$.

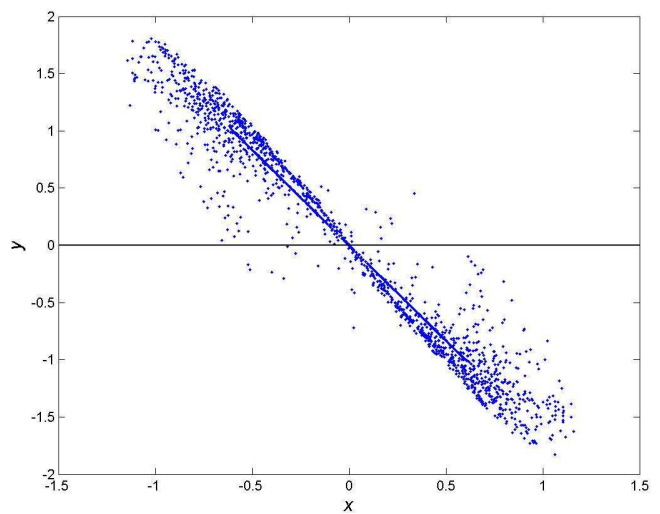

Fig. 15: Chaotic attractor of (7)-(8) when $r_{1}=r_{2}=1.2$ and $t \in$ $[0,300]$. 


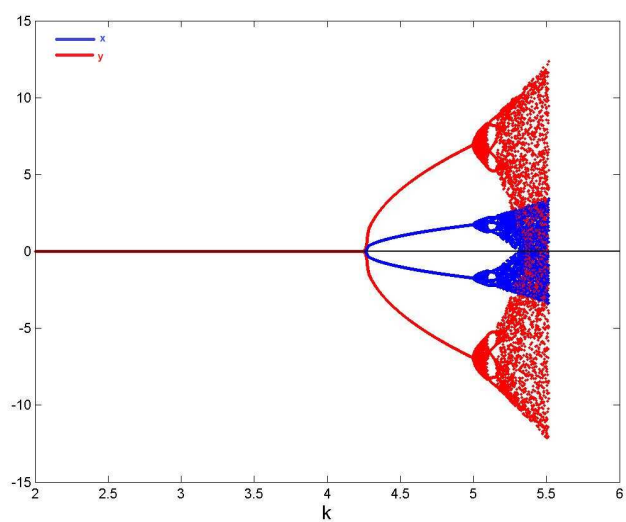

Fig. 16: Bifurcation diagram of (7)-(8) when $r_{1}=r_{2}=0.5$ and $t \in[0,300]$.

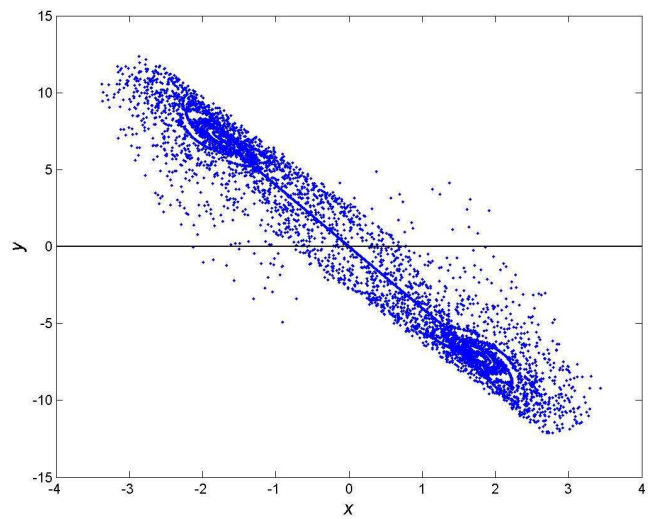

Fig. 17: Chaotic attractor of (7)-(8) when $r_{1}=r_{2}=0.5$ and $t \in$ $[0,300]$.

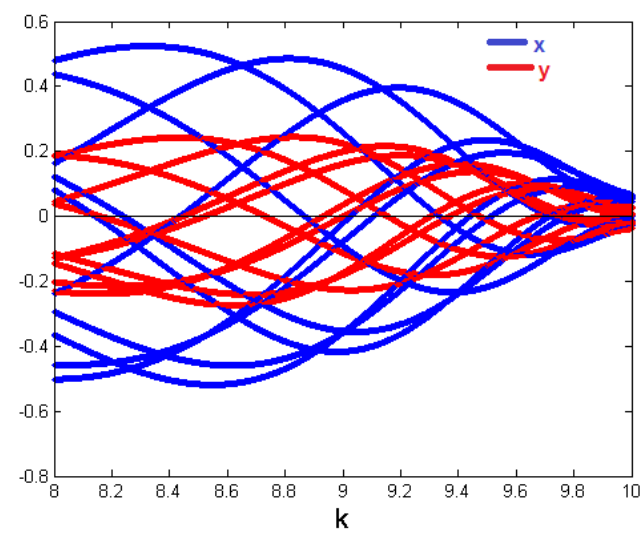

Fig. 18: Bifurcation of (7)-(8) when $r_{1}=0.35, r_{2}=0.20$ and $t \in[0,200]$.

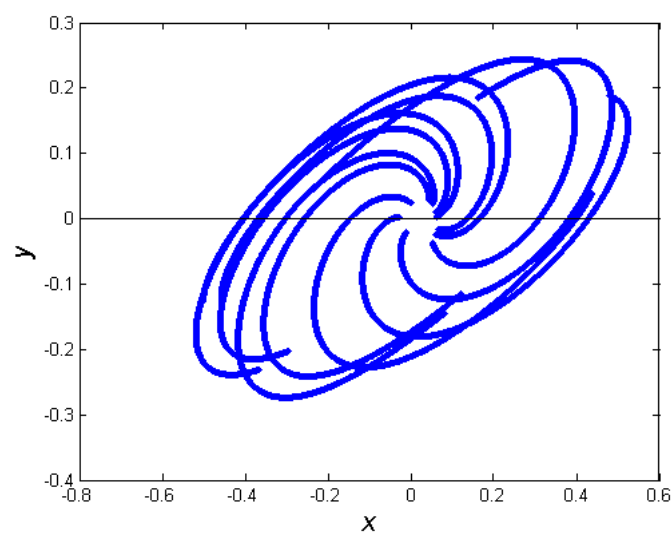

Fig. 19: Chaotic attractor of (7)-(8) when $r_{1}=0.35, r_{2}=0.20$ and $t \in[0,200]$.

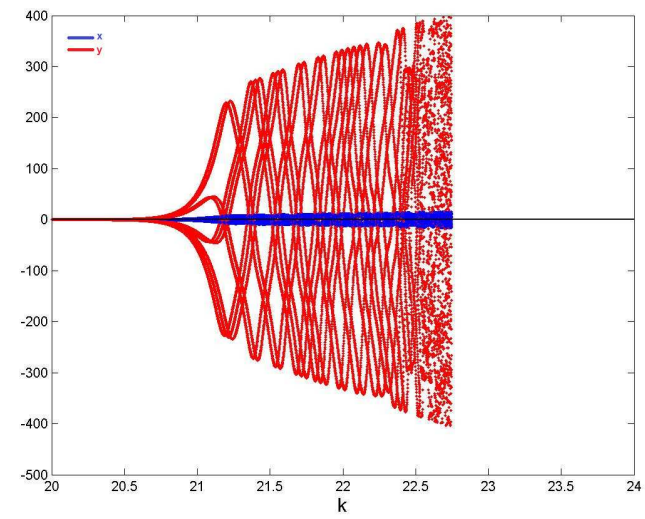

Fig. 20: Bifurcation of (7)-(8) when $r_{1}=0.05, r_{2}=0.1$ and $t \in$ $[0,200]$.

and the bifurcation is shown in Figure (18).

Take $r_{1}=0.35, r_{2}=0.20$ and $t \in[0,200]$ in (7)-(7) (Figures (18)-(19)).

- Case 3: $r_{1}<r_{2}$

Let $r_{1}<r_{2}$ and vary $k$ from 20 to 23 with step length 0.001 . The initial state of the system is again as above. The bifurcation occurs at $k=20.6$ and chaos shows at $k=22.5$.

Take $r_{1}=0.05, r_{2}=0.1$ and $t \in[0,200]$ in (7)-(8) (Figure (20)-(21)).

Take $r_{1}=0.15, r_{2}=0.20$ and $t \in[0,200]$ in (7)-(8) (Figure (22)-(23)). 


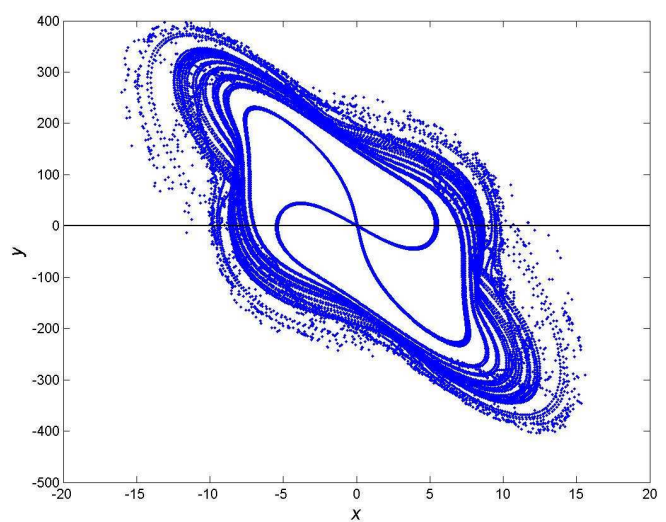

Fig. 21: Chaotic attractor of (7)-(8) when $r_{1}=0.05, r_{2}=0.1$ and $t \in[0,200]$.

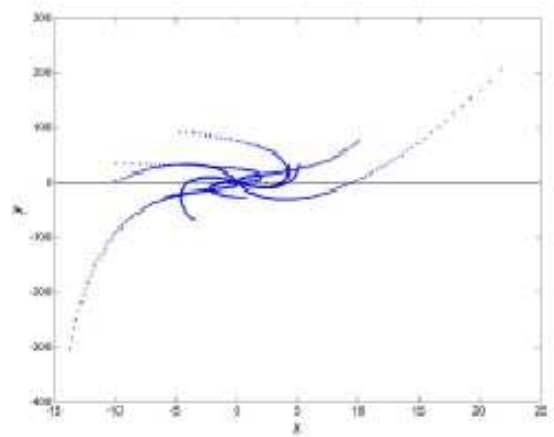

Fig. 22: Bifurcation of (7)-(8) when $r_{1}=0.15, r_{2}=0.20$ and $t \in[0,200]$.

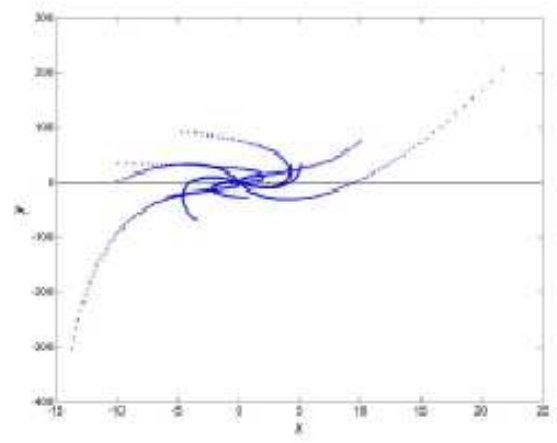

Fig. 23: Chaotic attractor of (7)-(8) when $r_{1}=0.15, r_{2}=0.20$ and $t \in[0,200]$.

\section{Conclusion}

Applying the semi-descretization process to the duffing system resulting in a discontinuous (sectionally continuous) dynamical system representing the duffing equations. Moreover, changing both the retardation parameter $r$ together with the time $t \in[0, T]$, has the strong effect on bifurcation, chaos, and chaotic attractor of the system. the discontinuous (sectionally continuous) dynamical system of the duffing model describes the dynamic Properties for different values of the retardation parameters $r_{1}$ and $r_{2}$ when the time is continuous. our analytical result ((11) and (12)) agrees with the numerical simulations. This shows the richness of the models of discontinuous (sectionally continuous) dynamical systems.

\section{References}

[1] N. A. Bohai, Continuous solutions of systems of nonlinear difference equations with continuous arguments and their properties, Journal of Nonlinear oscillations, 10, (2007).

[2] S. N. Elaydi, An Introduction to Difference Equations, Third Edition, Undergraduate Texts in Mathematics, Springer, New York, (2005).

[3] Jose'H. da Cruze, Pla'cido Z. Ta'boas, Periodic solutions and stability for a singularly perturbed linear delay differential equation, Journal of nonlinear analysis, 67, 1657-1667 (2007).

[4] A. El-Sayed, A. El-Mesiry and H. EL-Saka, On the fractional-order logistic equation, Applied Mathematics Letters, 20, 817-823 (2007).

[5] A. M. A. El-Sayed and M. E. Nasr, Existence of uniformly stable solutions of nonau- tonomous discontinuous dynamical systems J. Egypt Math. Soc., 19, (2011).

[6] A. M. A. El-Sayed and M. E. Nasr, On some dynamical properties of discontinuous dynamical systems, American Academic and Scholarly Research Journal, 2, 28-32 (2012).

[7] A. M. A. El-Sayed and M. E. Nasr, Dynamic properties of the predator-prey discontinuous dynamical system, Z . Naturforsch. A, 67a, 57-60 (2012).

[8] A. M. A. El-Sayed and M. E. Nasr, On some dynamical properties of the discontinuous dynamical system presents the Logistic equation with different delays, i-managers Journal on Mathematics, l, January - March (2012).

[9] A. M. A. El-Sayed and M. E. Nasr, Some Dynamic Properties of a Discontinuous Dynamical System, Alexandria Journal of Mathematics, 3, November (2012).

[10] A. M. A. El-Sayed and M. E. Nasr, Discontinuous Dynamical Systems and Fractional-Orders Difference Equations, Journal of Fractional, Calculus and Applications, 4, 1-9 Jan (2013).

[11] A.M.A. El-Sayed and S. M. Salman, Chaos and bifurcation of discontinuous dynamical sys- tems with piecewise constant arguments, Malaya Journal of Matematik, 1, 1519 (2012). 
[12] A.M.A. El-Sayed and S. M. Salman, Discontinuous dynamical systems generated by a semi-discretization process, Electronic Journal of Mathematical Analysis and Applications, 1, 1-8 Jan. (2013).

[13] J. Hale, Theory of functional differential equations, springr verlag, New York, (1977).

[14] Z. J. Jing and J. Yang, Chaos Solitons Fractals, 27, 259 (2006).

[15] X. Liu and D. Xiao, Chaos Solitons Fractals, 32, 80 (2007).

[16] Kuznetsov Yu.A., Elements of Applied Bifurcation Theory, Springer, 3rd edition, (2004). 